\title{
Enhanced Inter-Vehicular Relative Positioning
}

\author{
Thomas Speth ${ }^{1}$, Raphael Riebl ${ }^{1}$, Thomas Brandmeier ${ }^{1}$, Christian Facchi ${ }^{1}$, Ali H. Al-Bayatti ${ }^{2}$ and Ulrich Jumar ${ }^{3}$
}

\begin{abstract}
Intelligent Transportation System (ITS) applications for integral and cooperative vehicle safety as well as some Advanced Driver Assistance Systems (ADASs) benefit from precise determination of relative positions between dynamic traffic objects. With conventional Global Navigation Satellite System (GNSS) measurements, e.g. using Global Positioning System (GPS), the required accuracy cannot be achieved. For this reason, an exchange of GNSS observations via Vehicular Ad-Hoc Network (VANET) is proposed in this paper. In particular, the European Inter-Vehicle Communication (IVC) protocol stack ITS-G5 is employed. With these exchanged GNSS observations, Differential GNSS (DGNSS) or Real-Time Kinematic (RTK) calculations provide a precise relative position vector. However, due to relative movement of traffic objects, this position vector becomes obsolete for increasing transmission delays. For this reason, a mitigating kinematic model is set up and validated experimentally. With respect to fixed RTK solutions, this kinematic model reduces the errors by an average of $61 \%$ compared to position calculations ignoring IVC latency.
\end{abstract}

Index Terms - vehicular ad hoc networks, intelligent transport system, satellite navigation system, positioning, real time kinematic, moving base

\section{INTRODUCTION}

Investigations by World Health Organization (WHO) motivated the proclamation of the present decade for road safety [1]. Current statistics exhibit annual numbers of over 1.2 million lethal road accidents and up to 50 million additional injuries [2], [3]. Lacking legislation might be one reason for these numbers, but even in regions with strict laws it is still a path of trial and tribulation towards 'Vision Zero', i.e. no more lethal road accidents. Within the European Union for example, there are 1.4 million injured and over 25,000 killed people in 2014 [4]. Even worse, the traffic density and thus the traffic complexity is increasing there. A lot of accidents are caused by wrong behaviour of vehicle drivers. Thus, Advanced Driver Assistance Systems (ADASs) and integral safety applications can help avoiding accidents or at least reduce the caused injuries [5].

Inter-Vehicle Communication (IVC) is thereby a major key for a new generation of ADASs, because it enables interactive approaches for sensor systems as well as collaborative vehicle acting [6]. In this research, we are focusing on the first aspect. Compared to conventional environmental sensors like Radar

\footnotetext{
${ }^{1}$ CARISSMA, Technische Hochschule Ingolstadt, Ingolstadt, Germany, email: \{thomas.speth, raphael.riebl, thomas.brandmeier, christian.facchi\}@thi.de

${ }^{2}$ Software Technology Research Laboratory, De Montfort University, Leicester, UK, email: alihmohd@dmu.ac.uk

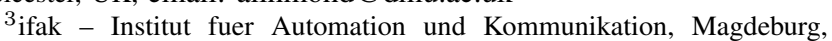
Germany, email: ulrich.jumar@ifak.eu
}

or Lidar (radio vs. light detection and ranging), IVC has two major benefits: There is no limiting opening angle, i.e. Vehicleto-Vehicle (V2V) communication acts like a $360^{\circ}$ field of view sensor. In addition, IVC can work under non-line-ofsight conditions albeit with reduced range. Other sensors are more prone to blind spots, e.g. at intersections.

When vehicles spread position data in their vicinity as specified by the Cooperative Awareness service [7], this information could be used for collision avoidance. However, such a use case requires mutual position knowledge with lane-level accuracy [8]. Standalone measurements using e.g. Global Positioning System (GPS) are too inaccurate for that purpose [9], but IVC is also an enabling technology for a Global Navigation Satellite System (GNSS) augmentation method called Cooperative Positioning (CP) [10].

Known methods for improved positioning are Differential GNSS (DGNSS) or Real-Time Kinematic (RTK). Both techniques lean on GNSS observation data stemming from a nearby reference station. Typically, an accuracy below $0.1 \mathrm{~m}$ root mean square is achievable with RTK. In a RTK setup, a moving GNSS receiver, called rover, calculates double differences from its own carrier phase measurements and the ones received from a reference station. The result of this calculation is a base line vector between both receivers. As the reference station's position is well-known, it is trivial to derive a highly accurate position of the rover in the global reference frame. [11, pp. 246 sqq.]

However, reference station operators often charge for these observation data and also the data link, often established by cellular mobile communication, causes costs. Hence, third-party reference stations are not available all the time or affordable in all regions. We propose as alternative to stationary reference stations so-called moving bases, which can be any other vehicle in the vicinity of the own egovehicle. This reduced RTK method requires no position coordinates from the moving base at the cost of not providing absolute positioning. Sending of mere GNSS observations suffices for calculating a base line vector. Future vehicle safety systems might benefit from this relative CP method. Similar approaches as those described in [12]-[14] deal with less accurate pseudorange measurements or do not adequately consider vehicle dynamics. The authors of [15] introduce a decentralized fusion center for cooperative multi-vehicle localization but rely on GNSS measurements for absolute positioning and environmental sensors for relative positioning.

With growing latency, however, the accuracy of the above mentioned relative $\mathrm{CP}$ method is decreasing because the base line vector is calculated between moving base and rover 


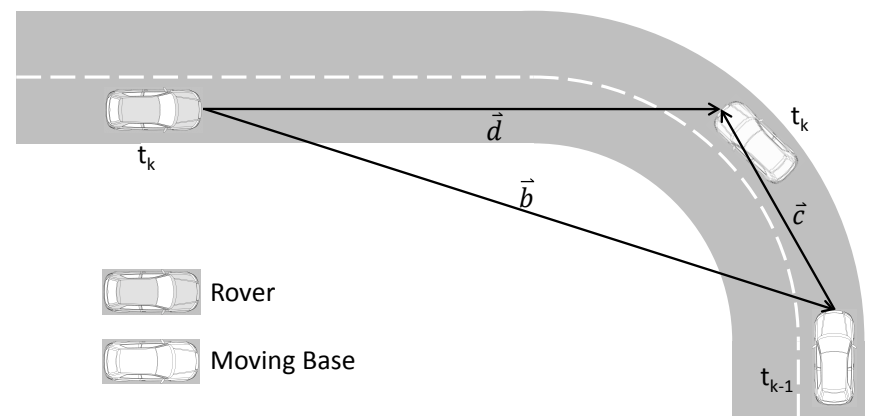

Fig. 1. Moving base sending data at time $t_{k-1}$ and a receiving rover

at the point in time of observation respectively. Thus, with larger differential age between both observation data, the calculated relative position vector becomes obsolete and inaccurate. Figure 1 shows this conjuncture exemplarily: Moving base makes observations at time $t_{k-1}$ and sends them afterwards. A rover receives these data and determines vector $\vec{b}$ using own current observations at time $t_{k}$. However, this vector has become obsolete, because the moving base has travelled the way $\vec{c}$ meanwhile, thus vector $\vec{d}$ gives the actual relative position between both at $t_{k}$. In this paper we analyse propagation delays and introduce a kinematic model for correcting this error source.

\section{CONCEPT OF Moving Base}

\section{A. Inter-Vehicle Data Exchange}

Inter-Vehicle Communication between rover and moving base is realised with the help of the European ITS-G5 protocol stack. Similar to existing Cooperative Awareness Messages (CAMs) [7], the IVC application for the proposed relative $\mathrm{CP}$ emits data packets at a rate of $10 \mathrm{~Hz}$. These packets contain vehicle dynamic information, such as acceleration and speed vectors, as well as GNSS observations. There are two main formats for encoding these GNSS observations: (a) Compact Measurement Record (CMR) [16] including its extensions CMR+ and CMRx [17] developed by Trimble. (b) The Radio Technical Commission for Maritime Services (RTCM) provides another widely used standard for DGNSS 10403.2 [18], which is considered hereinafter. But instead of sending a complete set of RTCM messages as recommended for full-fledged RTK, we send a reduced set comprising only GPS and GLONASS observations (RTCM messages 1004 and 1012). Discarded RTCM message types for RTK contain static data, e.g. antenna reference point, which are of no benefit for a moving base.

Transmission relies on the Single Hop Broadcast (SHB) mode of GeoNetworking [19], the network layer in the ITS-G5 communication architecture [20]. SHB is beneficial regarding the end-to-end latency between rover and moving base because it involves less queues and buffers compared to more sophisticated GeoNetworking modes like GeoBroadcast. In fact, except for the transmission queues within network interface cards, only queues regulating packet flow according to Decentralized Congestion Control (DCC) rules [21] are

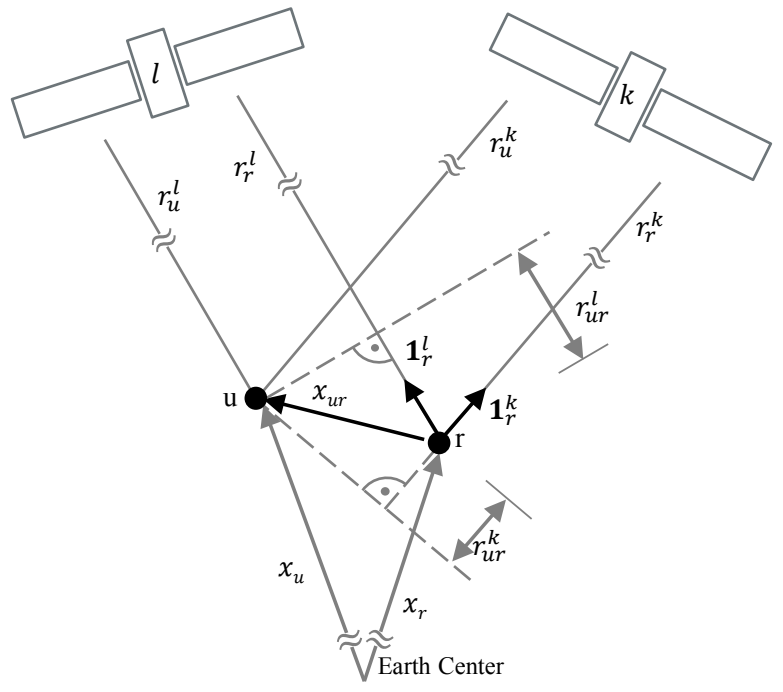

Fig. 2. Geometry of double difference measurements [11, p. 249]

found at transmitter side. Since only two vehicles are involved in the following test setup, DCC does not impose any transmission delays because both vehicles operate in a relaxed channel characterized by a low channel busy ratio. Thus, DCC will just pass packets from network through to access layer immediately. On receiver side, there are no delays imposed except for the processing time required for decoding a packet.

\section{B. Kinematic Model}

The backbone of relative positioning calculations are GNSS code measurements $\rho$ in units of length (1) and phase measurements $\varphi$ in cycles (2) [11, p. 238].

$$
\begin{aligned}
& \rho=r+I+T+c\left(\delta t_{u}-\delta t^{s}\right)+\varepsilon_{\rho} \\
& \varphi=\lambda^{-1}[r-I+T]+f \cdot\left(\delta t_{u}-\delta t^{s}\right)+N+\varepsilon_{\varphi}
\end{aligned}
$$

Thereby, $r$ denotes the geometric true range between satellite and receiver. Symbols $f$ and $\lambda$ are the frequency and wavelength of the GNSS signal, respectively. The terms include propagation delays through ionosphere $I$ and troposphere $T$ as well. $\delta t_{u}$ and $\delta t^{s}$ multiplied with the speed of light in vacuum $c$ represent receiver and satellite clock biases. The integer ambiguity of the constantly repeated code pattern is given by $N$. Other measurements and modelling errors are conflated in $\varepsilon$.

Dynamic traffic objects operating as moving base spread their observations via IVC. Any other vehicle receiving such observations encoded in RTCM data sets can process them along with own observation and thus determine the relative position between both observation locations. In a first processing step, single differences of the carrier phase measurements from satellite $k$ between a moving base and the ego-vehicle are formed (3) to eliminate nuisance parameters. Index $r$ indicates the moving base and index $u$ indicates the rover, i.e. the ego-vehicle. [11, pp. 242 sqq.] 


$$
\begin{aligned}
\varphi_{u r}^{k} & =\varphi_{u}^{k}-\varphi_{r}^{k}= \\
& =\lambda^{-1}\left[\left(r_{u}^{k}-r_{r}^{k}\right)-\left(I_{u}^{k}-I_{r}^{k}\right)+\left(T_{u}^{k}-T_{r}^{k}\right)\right]+ \\
& +f \cdot\left(\delta t_{u}-\delta t_{r}\right)+\left(N_{u}^{k}-N_{r}^{k}\right)+\left(\varepsilon_{\varphi, u}^{k}-\varepsilon_{\varphi, r}^{k}\right)= \\
& =\lambda^{-1}\left[r_{u r}^{k}-I_{u r}^{k}+T_{u r}^{k}\right]+f \cdot \delta t_{u r}+N_{u r}^{k}+\varepsilon_{\varphi, u r}^{k}
\end{aligned}
$$

Since common-mode errors vary slowly over time and influence all receivers in a certain area in equal measure, the differences of ionospheric errors $I_{u r}^{k}$ and tropospheric errors $T_{u r}^{k}$ can be dropped. Out of the single differences from satellite $k$ and $l$, double differences are formed as illustrated in Figure 2 to eliminate clock biases.

$$
\begin{aligned}
\varphi_{u r}^{k l} & =\varphi_{u r}^{k}-\varphi_{u r}^{l}= \\
& =\lambda^{-1} \cdot r_{u r}^{k l}+N_{u r}^{k l}+\varepsilon_{\varphi, u r}^{k l} \\
\text { with } r_{u r}^{k l} & =\left(r_{u}^{k}-r_{r}^{k}\right)-\left(r_{u}^{l}-r_{r}^{l}\right)=-\left(\mathbf{1}_{r}^{k}-\mathbf{1}_{r}^{l}\right) \cdot\left|\vec{x}_{u r}\right|
\end{aligned}
$$

Expanding this context to all $K$ satellites in view and taking satellite with index 1 as reference, Equation (5) with $(K-1)$ double differences can be formulated.

$$
\begin{gathered}
{\left[\begin{array}{c}
\varphi_{u r}^{(21)} \\
\varphi_{u r}^{(31)} \\
\vdots \\
\varphi_{u r}^{(K 1)}
\end{array}\right]=\lambda^{-1}\left[\begin{array}{c}
-\left(\mathbf{1}_{r}^{(2)}-\mathbf{1}_{r}^{(1)}\right)^{T} \\
-\left(\mathbf{1}_{r}^{(3)}-\mathbf{1}_{r}^{(1)}\right)^{T} \\
\vdots \\
-\left(\mathbf{1}_{r}^{(K)}-\mathbf{1}_{r}^{(1)}\right)^{T}
\end{array}\right]\left|\vec{x}_{u r}\right|+} \\
{\left[\begin{array}{c}
N_{u r}^{(21)} \\
N_{u r}^{(31)} \\
\vdots \\
N_{u r}^{(K 1)}
\end{array}\right]+\left[\begin{array}{c}
\varepsilon_{\varphi}^{(21)}, u r \\
\varepsilon_{\varphi}^{(31)} \\
\vdots \\
\varepsilon_{\varphi, u r}^{(K 1)}
\end{array}\right]}
\end{gathered}
$$

There are well known methods to solve the remaining integer ambiguities $N_{u r}^{(k 1)}$ and thus to determine $x_{u r}$, e.g. the least-squares ambiguity decorrelation adjustment (LAMBDA) [22], [23]. According to the confidence level of the solution, integer ambiguities are approximated as floats (float solution) or fixed to integers (fixed solution). The wanted base line vector $\vec{b}$ equals then $\left(-\vec{x}_{u r}\right)$.

However, these calculations might rest on obsolete observations and are thus inappropriate for an approach with moving bases where IVC latencies occur. For this reason, we use additional information, i.e. driving dynamic data. A moving base sends these data as specified by ETSI [7] along with RTCM data. A rover can then estimate a moving base's travelled distance while GNSS observations are transmitted via IVC as follows.

$$
\begin{gathered}
{\left[\begin{array}{l}
\Delta x_{t_{k}} \\
\Delta y_{t_{k}}
\end{array}\right]=\left[\begin{array}{l}
v_{x, t_{k-1}} \\
v_{y, t_{k-1}}
\end{array}\right] \Delta t+\frac{1}{2}\left[\begin{array}{l}
a_{x, t_{k-1}} \\
a_{y, t_{k-1}}
\end{array}\right](\Delta t)^{2}} \\
\text { with } \Delta t=t_{k}-t_{k-1} \\
\vec{c}=\left[\begin{array}{c}
\Delta E_{t_{k}} \\
\Delta N_{t_{k}}
\end{array}\right]=\left[\begin{array}{cr}
\cos \psi_{t_{k-1}} & -\sin \psi_{t_{k-1}} \\
\sin \psi_{t_{k-1}} & \cos \psi_{t_{k-1}}
\end{array}\right]\left[\begin{array}{l}
\Delta x_{t_{k}} \\
\Delta y_{t_{k}}
\end{array}\right] \\
\vec{d}=\vec{b}+\vec{c}
\end{gathered}
$$

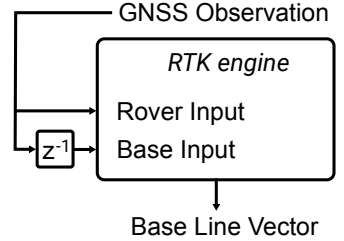

(a)

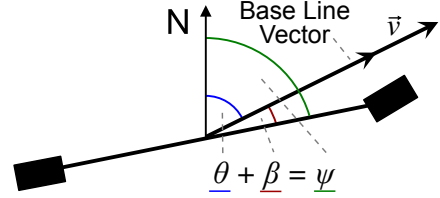

(b)
Fig. 3. Precise yaw angle determination using (a) RTK heading based on delayed observations as base input and (b) angles from a single-track-model

Thereby, $x$ and $y$ are coordinates in moving base's body frame [24], where $x$ is in vehicular longitudinal and $y$ in lateral direction. A rover receives the data at time $t_{k}$, which a moving base has sent at time $t_{k-1}$. Since a rover has no information about moving base after time $t_{k-1}$, a constant acceleration is assumed for time $\Delta t$. Therefore, a statistic estimator such as Kalman filter [25] is not applicable. Because of traffic's dynamic, a distinct neighbouring vehicle is only shortly in the role of a moving base, limited by the time it is within SHB communication range. This might be too short in many scenarios for proper filter initialization. Additionally, estimations are conducted at rover side and would require transmission of a plethora of moving base parameters via IVC, which leads to increased channel load. Determination of $\Delta t$ involves no further communication overhead then again, because RTCM message 1004 sent by a moving base contains already GPS time of week in milliseconds. This time stamp can be subtracted from current time provided by the rover's GNSS receiver. Finally, the estimated distance is transformed to east-north-up (ENU) reference frame as given by Equation (7), where $\psi$ denotes the yaw angle.

Velocities and accelerations can be measured quite well with sensors available in vehicles nowadays. However, it remains a challenge to determine the yaw angle $\psi$ accurately, i.e. the vehicle's longitudinal orientation compared to north. Thereby, we are using modified RTK calculation as depicted in Figure 3. Different to the original procedure, we buffer previous observation data for $0.1 \mathrm{~s}$ and use them as correction data input. By this procedure, a moving base can calculate a base line vector that corresponds to the own travelled way in ENU reference frame. Thus, we get the heading angle $\theta$ compared to north. Without loss of generality, the GNSS antenna is assumed to be mounted at vehicle's vertical axis, i.e. above vehicle's center of gravity. The difference between driving direction $\theta$ and vehicle's longitudinal direction is the slip angle, denoted as $\beta$ in Equation (9). This angle can be calculated with the help of a single-track-model in state space representation as follows [26].

$$
\begin{gathered}
{\left[\begin{array}{c}
\ddot{\psi} \\
\dot{\beta}
\end{array}\right]=\left[\begin{array}{cc}
-\frac{c_{f} l_{f}^{2}+c_{r} l_{r}^{2}}{v \Theta} & -\frac{c_{f} l_{f}-c_{r} l_{r}}{\Theta} \\
-1-\frac{c_{f} l_{f}-c_{r} l_{r}}{v^{2} m} & \frac{c_{f} c_{r}}{v m}
\end{array}\right]\left[\begin{array}{c}
\dot{\psi} \\
\beta
\end{array}\right]+} \\
+\left[\begin{array}{c}
\frac{c_{r} l_{r}}{\Theta} \\
\frac{c_{f}}{v m}
\end{array}\right] \delta \\
\psi_{t_{k-1}}=\theta_{t_{k-1}}+\beta_{t_{k-1}} \\
\psi_{t_{k}}=\psi_{t_{k-1}}+\dot{\psi}_{t_{k-1}} \cdot \Delta t
\end{gathered}
$$




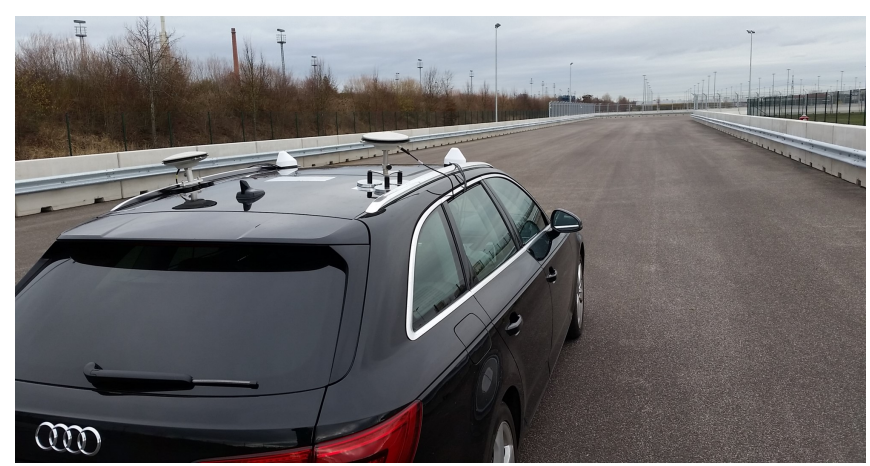

Fig. 4. Test vehicle with mounted moving base and rover modules on CARISSMA test track

Cornering stiffness at front and rear axle are denoted by $c_{f}$ and $c_{r}$, respectively. The mass $m$ of the vehicle is centred in the gravity point, which has a horizontal distance of $l_{r}$ from rear axle and $l_{f}$ from front axle. The moment of inertia is given by $\Theta$. The model's input is the steering angle $\delta$ of the front wheels. All values are given in body frame coordinates.

\section{EXPERIMENTAL EVALUATION}

\section{A. Test setup}

For experimental evaluation, moving base and rover modules were mounted on top of one vehicle. As shown in Figure 4, the moving base's GNSS and IVC antennas were put on the left side, the rover's antennas on the right side of the roof. Major benefit of this setup is the always well known ground truth, i.e. the distance between both GNSS antenna centres is fixed to $0.88 \mathrm{~m}$. While radio propagation effects are neglected by this setup, the end-to-end latency induced by hard- and software processing can still be studied. Each module consists of a Topcon Euro-112 PII GNSS receiver board, a NovAtel GPS-702-GG antenna and a Cohda MK3 IVC On-Board Unit (OBU). Additionally, the software package RTKLIB was used [27].

Experiments were conducted on different roads. On the CARISSMA test track, the velocity of $30 \mathrm{~km} / \mathrm{h}$ was low. But by driving tight circles and figures of eight, these tests exhibit highest lateral dynamics. Further tests were carried out on real roads to consider authentic driving dynamics, i.e. on country roads including driving through villages and towns as well as on highway. The velocity on country roads was up to $100 \mathrm{~km} / \mathrm{h}$ and on highway between $80 \mathrm{~km} / \mathrm{h}$ to $180 \mathrm{~km} / \mathrm{h}$. Measurements were sampled with $10 \mathrm{~Hz}$.

\section{B. Positioning Results}

Table I shows 50th (median), 90th and 95th percentile of each test run. These percentiles are given for the existing method (original) and the improved moving base (correction) taking latency and driving dynamic into account. Furthermore, these results are provided for both positioning solutions, i.e. fixed and float solution. The ratio of both solutions per test run is given in the last column. These numbers do not necessarily sum up to $100 \%$ because sometimes no RTK solution could be calculated at all. Fortunately, this happens in less than

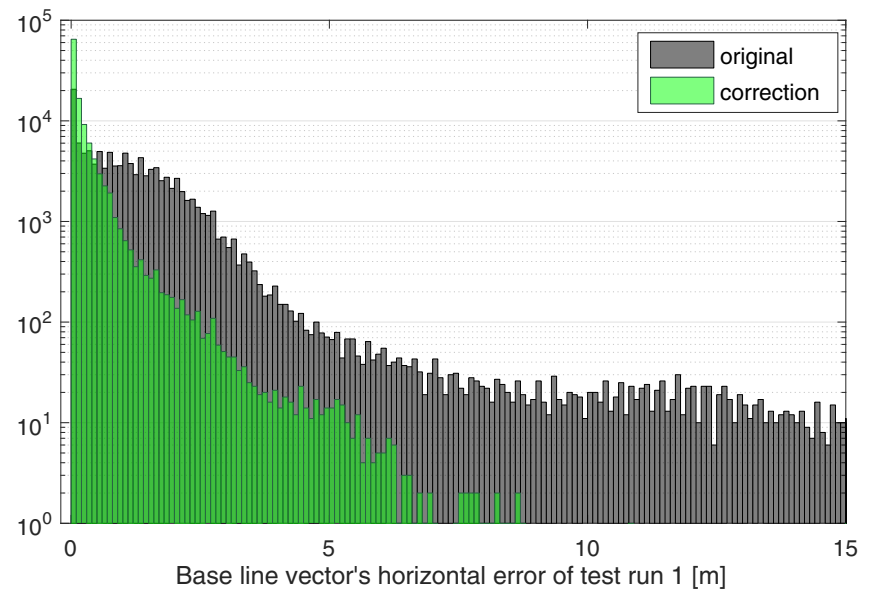

Fig. 5. Histogram of horizontal errors

$5 \%$ of all positions calculations. The grey column highlights the achieved accuracy levels. In addition to Table I, Figure 5 shows an error histogram of test run 1 representatively for all test runs.

Though our approach does not reach the accuracy of a stationary reference base, the medians for fixed solutions are better than half a metre in most test runs. Thus, our introduced method seems to be applicable for cooperative safety systems, e.g. autonomous emergency braking or just for collision warning. Also float solutions can be used for these use cases because they are within lane level accuracy. Compared to conventional moving base calculations, position accuracy is improved by an average of $58 \%$ over all test runs. In summary, a RTK solution is calculable most of the time and highly accurate.

The solutions are also improved when focusing on the 90th and 95th percentile. However, there is still considerable scattering up to a few metres causing some loss in precision due to highly deviating positioning solutions.

\section{Observed Latency}

Figure 6 depicts the measured latencies for each test run. These measurements cover the whole RTCM packet lifetime starting with generation by moving base's application and finishing with rover's application processing. Beside the end-to-end communication delay, these latencies thus also account for applications' processing time. Except for test run 2, data shows that latency varies between $60 \mathrm{~ms}$ to $100 \mathrm{~ms}$ as desired for the kinematic model. However, in future real world IVC scenarios, there could occur higher and more varying latencies, because other IVC services with possibly higher priority compete for channel access with our described DGNSS service.

Simulations based on the INET framework ${ }^{1}$ give some insights about delays caused by channel access when not just one but a multitude of transmitting vehicles are in close vicinity. In our simulation model, each vehicle tries to transmit two packets of 1000 bytes length every $100 \mathrm{~ms}$, mimicking CAM

${ }^{1}$ INET 3.2.4, https://inet. omnetpp.org 
TABLE I

PERCENTILES OF HORIZONTAL ERRORS

\begin{tabular}{|c|c|c|c|c|c|c|c|c|c|}
\hline & \multirow[b]{2}{*}{ road } & \multirow[b]{2}{*}{ solution } & \multicolumn{2}{|c|}{ P50 } & \multicolumn{2}{|c|}{ P90 } & \multicolumn{2}{|c|}{ P95 } & \multirow[b]{2}{*}{ ratio } \\
\hline & & & original & correction & original & correction & original & correction & \\
\hline \multirow[t]{2}{*}{ Run 1} & \multirow[t]{2}{*}{ Highway } & float & $0.95 \mathrm{~m}$ & $0.22 \mathrm{~m}$ & $3.05 \mathrm{~m}$ & $0.77 \mathrm{~m}$ & $3.93 \mathrm{~m}$ & $1.36 \mathrm{~m}$ & $43 \%$ \\
\hline & & fixed & $0.92 \mathrm{~m}$ & $0.08 \mathrm{~m}$ & $2.57 \mathrm{~m}$ & $0.90 \mathrm{~m}$ & $3.30 \mathrm{~m}$ & $0.91 \mathrm{~m}$ & $54 \%$ \\
\hline \multirow[t]{2}{*}{ Run 2} & \multirow[t]{2}{*}{ Highway } & float & $1.03 \mathrm{~m}$ & $0.47 \mathrm{~m}$ & $2.13 \mathrm{~m}$ & $1.36 \mathrm{~m}$ & $2.44 \mathrm{~m}$ & $1.71 \mathrm{~m}$ & $72 \%$ \\
\hline & & fixed & $0.31 \mathrm{~m}$ & $0.16 \mathrm{~m}$ & $1.28 \mathrm{~m}$ & $0.92 \mathrm{~m}$ & $1.65 \mathrm{~m}$ & $1.28 \mathrm{~m}$ & $28 \%$ \\
\hline \multirow[t]{2}{*}{ Run 3} & \multirow[t]{2}{*}{ Highway } & float & $2.12 \mathrm{~m}$ & $0.72 \mathrm{~m}$ & $4.24 \mathrm{~m}$ & $2.27 \mathrm{~m}$ & $6.48 \mathrm{~m}$ & $2.96 \mathrm{~m}$ & $70 \%$ \\
\hline & & fixed & $1.36 \mathrm{~m}$ & $0.41 \mathrm{~m}$ & $3.80 \mathrm{~m}$ & $1.80 \mathrm{~m}$ & $5.70 \mathrm{~m}$ & $2.56 \mathrm{~m}$ & $28 \%$ \\
\hline \multirow{2}{*}{ Run 4} & Country & float & $0.32 \mathrm{~m}$ & $0.22 \mathrm{~m}$ & $1.53 \mathrm{~m}$ & $1.32 \mathrm{~m}$ & $1.92 \mathrm{~m}$ & $1.80 \mathrm{~m}$ & $39 \%$ \\
\hline & Road & fixed & $0.21 \mathrm{~m}$ & $0.09 \mathrm{~m}$ & $0.94 \mathrm{~m}$ & $0.59 \mathrm{~m}$ & $1.25 \mathrm{~m}$ & $0.87 \mathrm{~m}$ & $60 \%$ \\
\hline \multirow{2}{*}{ Run 5} & Country & float & $1.33 \mathrm{~m}$ & $0.44 \mathrm{~m}$ & $4.85 \mathrm{~m}$ & $1.60 \mathrm{~m}$ & $6.43 \mathrm{~m}$ & $2.31 \mathrm{~m}$ & $68 \%$ \\
\hline & Road & fixed & $0.17 \mathrm{~m}$ & $0.06 \mathrm{~m}$ & $2.38 \mathrm{~m}$ & $0.90 \mathrm{~m}$ & $3.69 \mathrm{~m}$ & $1.58 \mathrm{~m}$ & $31 \%$ \\
\hline \multirow[t]{2}{*}{ Run 6} & Country & float & $0.67 \mathrm{~m}$ & $0.26 \mathrm{~m}$ & $3.23 \mathrm{~m}$ & $1.12 \mathrm{~m}$ & $4.87 \mathrm{~m}$ & $1.65 \mathrm{~m}$ & $53 \%$ \\
\hline & Road & fixed & $0.53 \mathrm{~m}$ & $0.28 \mathrm{~m}$ & $2.93 \mathrm{~m}$ & $1.72 \mathrm{~m}$ & $4.49 \mathrm{~m}$ & $2.61 \mathrm{~m}$ & $46 \%$ \\
\hline \multirow[t]{2}{*}{ Run 7} & Country & float & $0.44 \mathrm{~m}$ & $0.31 \mathrm{~m}$ & $2.10 \mathrm{~m}$ & $1.28 \mathrm{~m}$ & $3.07 \mathrm{~m}$ & $1.91 \mathrm{~m}$ & $54 \%$ \\
\hline & Road & fixed & $0.27 \mathrm{~m}$ & $0.08 \mathrm{~m}$ & $2.38 \mathrm{~m}$ & $0.69 \mathrm{~m}$ & $3.62 \mathrm{~m}$ & $1.15 \mathrm{~m}$ & $46 \%$ \\
\hline \multirow[t]{2}{*}{ Run 8} & Test & float & l & I & I & I & I & I & $0 \%$ \\
\hline & Track & fixed & $0.21 \mathrm{~m}$ & $0.13 \mathrm{~m}$ & $1.02 \mathrm{~m}$ & $1.09 \mathrm{~m}$ & $1.71 \mathrm{~m}$ & $1.72 \mathrm{~m}$ & $99 \%$ \\
\hline
\end{tabular}

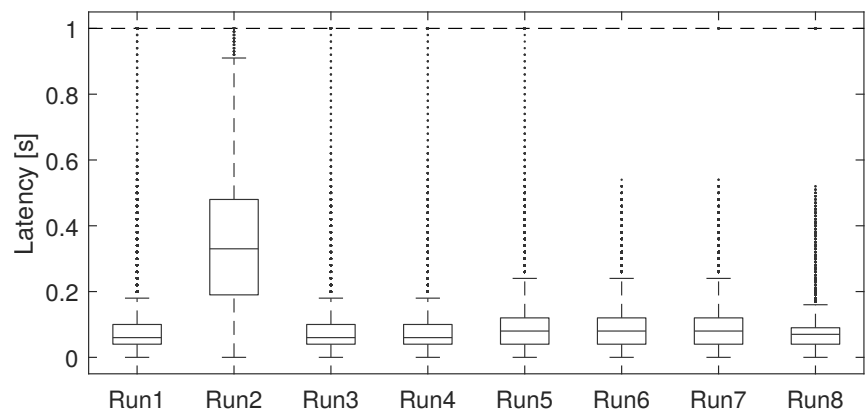

Fig. 6. Latency box plots of each test run

and RTCM packets. All vehicles are within communication range mutually, i.e. there are no hidden stations. INET's simulated network interface cards are configured to behave according to IEEE 802.11 for Vehicular Ad-Hoc Networks (VANETs). Just as with SHB, each packet is broadcasted with a bitrate of $6 \mathrm{Mbps}$. Simulation results presented in Figure 7 show no significant latency up to 32 vehicles. Mean latency impairs when the number of vehicles is increased further because channel's capacity allows only for about 660 packets per second, i.e. roughly 33 vehicles in this simulation setup. Consequently, the kinematic model's demand for low latencies justifies the need for proper management of available channel capacity, i.e. congestion control such as DCC.

\section{CONCLUSION AND FurTher WORK}

The introduced method suggests to send GNSS observation and driving dynamic data via IVC based on VANET technology. A kinematic model calculates the position relative to the sending vehicle out of received and own data. The presented results indicate that this kind of IVC application can act like a sensor for accurate relative positioning without the limitations of conventional environmental sensors. The

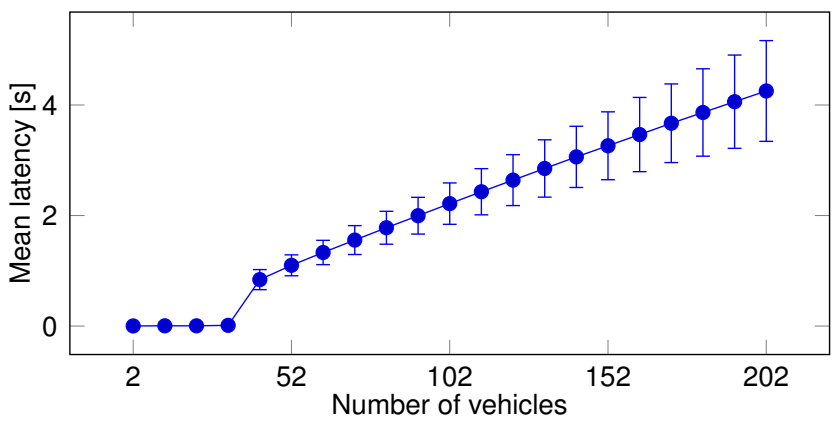

Fig. 7. Channel access delays (mean and $\pm \sigma$ )

measurements are on average within lane level accuracy, but exhibit large variances. Thus, the measurements are accurate but not precise. Improving the calculation confidence is hence a task for future work. Generally, it is recommended that moving bases also send confidence values of their driving dynamic sensors for determining confidence levels.

Furthermore, it has to be mentioned that for RTK calculations at least five common satellites at base and rover side have to be within the field of view. Especially in urban canyons the sky view can be restricted and thus the number of satellites in the field of view decreased. With new GNSSs like the European Galileo or regional positioning systems like the Japanese QZSS there will be more satellites receivable at user's location.

Another challenge is the initialisation time of RTK calculation. Nowadays, GNSS receivers generate a first RTK position fix after about $10 \mathrm{~s}$. Considering the IVC communication range, this time interval might be too long, i.e. dangerous traffic scenarios might occur before a base line vector has been calculated. Future developments can focus either on reducing RTK initialisation time or on extending communication range, e.g. by using multi-hop packet forwarding. The latter 
approach, however, needs additional investigations regarding communication performance.

\section{ACKNOWLEDGMENT}

The authors would like to thank AUDI AG for supporting their research.

\section{REFERENCES}

[1] WHO. (2009). Global status report on road safety, [Online]. Available: http : / / www . who . int / violence _ injury _ prevention / road _ safety - status / 2009 / en/ (visited on 03/18/2016).

[2] — , (2013). Global status report on road safety 2013, [Online]. Available: http : / / www . who . int / violence _ injury - prevention / road_safety_status/2013/en/ (visited on 03/30/2016).

[3] —, (2015). Global status report on road safety 2015, [Online]. Available: http : / / www . who . int / violence _ injury - prevention / road_safety_status/2015/en/ (visited on 03/30/2016).

[4] European Commission. (2015). Statistics - accidents data, 1995-2015, [Online]. Available: http : / / ec. europa.eu/transport/road_safety / specialist / statistics / index_en . htm (visited on 06/23/2015).

[5] W. Louwerse and S. Hoogendoorn, "ADAS safety impacts on rural and urban highways," in IEEE Intelligent Vehicles Symposium, Jun. 2004, pp. 887-890.

[6] J. Huang and H.-S. Tan, "Error analysis and performance evaluation of a future-trajectory-based cooperative collision warning system," IEEE Trans. Intell. Transp. Syst., vol. 10, no. 1, pp. 175-180, 2009.

[7] ETSI, Intelligent Transport Systems (ITS); Vehicular Communications; Basic Set of Applications; Part 2: Specification of Cooperative Awareness Basic Service, EN 302 637-2 V1.3.2, European Telecommunications Standards Institute, Nov. 2014.

[8] S. E. Shladover and S.-K. Tan, "Analysis of vehicle positioning accuracy requirements for communicationbased cooperative collision warning," Journal of Trans. Intell. Transp. Syst., vol. 10, no. 3, pp. 131-140, 2006.

[9] J. G. Grimes, "Global positioning system - standard positioning service - performance standard," 2008.

[10] N. Alam and A. Dempster, "Cooperative positioning for vehicular networks: Facts and future," IEEE Transactions on Intelligent Transportation Systems, vol. 14, no. 4, pp. 1708-1717, Dec. 2013.

[11] P. Misra and P. Enge, Global positioning system: Signals, measurements, and performance. Lincoln, Mass: Ganga-Jamuna Press, 2006.

[12] K. Ansari, C. Wang, L. Wang, and Y. Feng, "Vehicleto-vehicle real-time relative positioning using $5.9 \mathrm{GHz}$ DSRC media," in Vehicular Technology Conference (VTC Fall), 2013 IEEE 78th, 2013.
[13] N. Alam, A. Tabatabaei Balaei, and A. Dempster, "Relative positioning enhancement in VANETs: A tight integration approach," IEEE Trans. Intell. Transp. Syst., vol. 14, no. 1, pp. 47-55, 2013.

[14] W. Travis and D. M. Bevly, "Trajectory duplication using relative position information for automated ground vehicle convoys," in Position, Location and Navigation Symposium, 2008 IEEE/ION, 2008, pp. 1022-1032.

[15] H. Li and F. Nashashibi, "Cooperative multi-vehicle localization using split covariance intersection filter," in 2012 IEEE Intelligent Vehicles Symposium (IV), Jun. 2012, pp. 211-216.

[16] N. Talbot, "Compact data transmission standard for high-precision GPS," in Proceedings of ION GPS, vol. 9, 1996, pp. 861-871.

[17] Trimble, "CMRx: A new correction format from Trimble," Trimble Navigation Limited, White Paper, 2009.

[18] RTCM, Differential GNSS (Global Navigation Satellite Systems) Services, RTCM 10403.2, Radio Technical Commission for Maritime Services, Feb. 2013.

[19] ETSI, Intelligent Transport Systems (ITS); Vehicular Communications; GeoNetworking; Part 4: Geographical addressing and forwarding for point-topoint and point-to-multipoint communications; Subpart 1: Media-Independent Functionality, EN 302 6364-1 V1.2.1, European Telecommunications Standards Institute, Jul. 2014.

[20] - Intelligent Transport Systems (ITS); Communications Architecture, EN 302665 V1.1.1, European Telecommunications Standards Institute, Sep. 2010.

[21] - Intelligent Transport Systems (ITS); Decentralized Congestion Control Mechanisms for Intelligent Transport Systems operating in the the $5 \mathrm{GHz}$ range; Access layer part, TS 102687 V1.1.1, European Telecommunications Standards Institute, Jul. 2011.

[22] P. J. G. Teunissen, "The least-squares ambiguity decorrelation adjustment: A method for fast GPS integer ambiguity estimation," Journal of Geodesy, vol. 70, no. 1, pp. 65-82, 1995.

[23] P. J. G. Teunissen, P. J. De Jonge, and C. C. J. M. Tiberius, "Performance of the LAMBDA method for fast GPS ambiguity resolution," Navigation, vol. 44, no. 3, pp. 373-383, 1997.

[24] ISO, Road vehicles - vehicle dynamics and roadholding ability - vocabulary, ISO 8855, International Organization for Standardization, 2011.

[25] R. E. Kalman, "A new approach to linear filtering and prediction problems," Journal of basic Engineering, vol. 82, no. 1, pp. 35-45, 1960.

[26] P. Riekert and T. E. Schunck, "Zur Fahrmechanik des gummibereiften Kraftfahrzeugs," Ingenieur-Archiv, vol. 11, no. 3, pp. 210-224, Jun. 1940.

[27] T. Takasu, RTKLIB: an open source program package for GNSS positioning, version 2.4.2, 2013. 